\title{
Mineral and bone disorder in Chinese dialysis patients: a multicenter study
}

Xianglei Kong ${ }^{1,26 \dagger}$, Luxia Zhang ${ }^{1 \dagger}$, Ling Zhang ${ }^{2}$, Nan Chen ${ }^{3}$, Yong Gu ${ }^{4}$, Xueqing Yu' ${ }^{5}$, Wenhu Liu ${ }^{6}$, Jianghua Chen ${ }^{7}$, Liren Peng ${ }^{8}$, Weijie Yuan ${ }^{9}$, Hua Wu ${ }^{10}$, Wei Chen ${ }^{11}$, Minhua Fan ${ }^{12}$, Liqun He ${ }^{13}$, Feng Ding ${ }^{14}$, Xiangmei Chen ${ }^{15}$, Zuying Xiong ${ }^{16}$, Jinyuan Zhang ${ }^{17}$, Qiang Jia ${ }^{18}$, Wei Shi ${ }^{19}$, Changying Xing ${ }^{20}$, Xiaoling Tang ${ }^{21}$, Fanfan Hou ${ }^{22}$, Guiyang Shu ${ }^{23}$, Changlin Mei ${ }^{24}$, Li Wang ${ }^{25}$, Dongmei Xu ${ }^{26}$, Zhaohui $\mathrm{Ni}^{27}$, Li Zuo ${ }^{1}$, Mei Wang ${ }^{1,28,29^{*}}$ and Haiyan Wang ${ }^{1}$

\begin{abstract}
Background: Mineral and bone disorder (MBD) in patients with chronic kidney disease is associated with increased morbidity and mortality. Studies regarding the status of MBD treatment in developing countries, especially in Chinese dialysis patients are extremely limited.

Methods: A cross-sectional study of 1711 haemodialysis (HD) patients and 363 peritoneal dialysis (PD) patients were enrolled. Parameters related to MBD, including serum phosphorus (P), calcium (Ca), intact parathyroid hormone (iPTH) were analyzed. The achievement of MBD targets was compared with the results from the Dialysis Outcomes and Practice Study (DOPPS) 3 and DOPPS 4. Factors associated with hyperphosphatemia were examined.

Results: Total 2074 dialysis patients from 28 hospitals were involved in this study. Only 38.5\%, 39.6\% and 26.6\% of them met the Kidney Disease Outcomes Quality Initiative (K/DOQI) defined targets for serum P, Ca and iPTH levels. Serum $P$ and $\mathrm{Ca}$ levels were statistically higher $(\mathrm{P}<0.05)$ in the HD patients compared with those of PD patients, which was $(6.3 \pm 2.1) \mathrm{mg} / \mathrm{dL}$ vs $(5.7 \pm 2.0) \mathrm{mg} / \mathrm{dL}$ and $(9.3 \pm 1.1) \mathrm{mg} / \mathrm{dL}$ vs $(9.2 \pm 1.1) \mathrm{mg} / \mathrm{dL}$, respectively. Serum iPTH level were statistically higher in the PD patients compared with those of HD patients $(P=0.03)$. The percentage of patients reached the $\mathrm{K} / \mathrm{DOQ}$ targets for $\mathrm{P}(37.6 \%$ vs $49.8 \%$ vs $54.5 \%, \mathrm{P}<0.01)$, Ca (38.6\% vs $50.4 \%$ vs $56.0 \%, \mathrm{P}<0.01)$ and $\mathrm{PTH}(26.5 \%$ vs $31.4 \%$ vs $32.1 \%, \mathrm{P}<0.01)$ were lower among HD patients, compared with the data from DOPPS 3 and DOPPS 4. The percentage of patients with serum phosphorus level above $5.5 \mathrm{mg} / \mathrm{dL}$ was $57.4 \%$ in HD patients and $47.4 \%$ in PD patients. Age, dialysis patterns and region of residency were independently associated with hyperphosphatemia.
\end{abstract}

Conclusions: Status of MBD is sub-optimal among Chinese patients receiving dialysis. The issue of hyperphosphatemia is prominent and needs further attention.

Keywords: End stage renal disease, Mineral and bone disorder, Epidemiology

\footnotetext{
*Correspondence: wangmei1949@163.com

${ }^{\dagger}$ Equal contributors

${ }^{1}$ Renal Division, Department of Medicine, Peking University First Hospital; Peking University Institute of Nephrology; Key Laboratory of Renal Disease, Ministry of Health of China; Key Laboratory of Chronic Kidney Disease Prevention and Treatment (Peking University), Ministry of Education, Beijing, China

${ }^{28}$ Department of Nephrology, Peking University People's Hospital, Beijing,

China

Full list of author information is available at the end of the article
} 


\section{Background}

Mineral and bone disorder (MBD) is a common complication of chronic kidney disease (CKD), and has been associated with increased risk of cardiovascular calcification, arterial dysfunction, morbidity and mortality $[1,2]$. Recently, increased attention has been focused on MBD, and hyperphosphatemia is a central role. A meta-analysis revealed that hyperphosphatemia is an independent risk factor for mortality among CKD patients [3]. Gradual decline in renal phosphorus clearance during the progression of CKD leads to an increase in serum phosphorus concentrations [4], which plays an important role in development of MBD [5]. Hyperphosphatemia may also contribute to vascular calcification and therefore higher risk of cardiovascular morbidity $[1,2]$.

The Kidney Disease Outcomes Quality Initiative (K/DOQI) clinical practice guidelines for MBD were released in 2003, aiming at assisting nephrologists in developing an integrated approach to the diagnosis and management of MBD. Since then, studies have suggested an improvement in treatment of MBD in many countries [6,7]. However, studies regarding the status of MBD treatment in developing countries are extremely limited, where the accessibility to healthcare resources and structure of health care system are different. Therefore, the Practice Patterns and Improvement Study of Bone Metabolism and Disease in patients with CKD (PPIS) was initiated to provide national data on the status of MBD treatment among patients receiving dialysis in China.

\section{Methods}

\section{Study population}

Twenty eight renal divisions from 9 provinces in China representing different geographic distribution and economic status were recruited as a convenience sample on a voluntary basis from January 2006 to June 2006. A map marked with study centers is provided in Figure 1. Adult ESRD patients receiving stable hemodialysis (HD) or peritoneal dialysis (PD) for at least 3 months were included in this study. Patients with renal tubule acidosis, multiple myeloma, idiopathic hypercalcemia or metastatic carcinoma of bone were excluded from the study. The ethics committee of Peking University First Hospital approved the study. All participants gave written informed consent prior to data collection.

\section{Data collection}

A questionnaire documenting information of sociodemographic status, personal and family health history, dialysis prescription, and medication for MBD were finished by doctors or nurses. Then a blood sample were drawn after an overnight fast of at least 8 hours, and before the HD session for HD patients. Parameters related to MBD, including serum phosphorus, calcium and intact parathyroid hormone concentration (iPTH) were measured at central laboratory of each study center. Serum phosphorus and serum calcium were measured by spectrophotometry assay. iPTH was measured by immunoradiometric or immunochemiluminometric assays. Corrected calcium was calculated as total calcium $(\mathrm{mg} / \mathrm{dL})+0.8 \times(4-$ serum albumin $(\mathrm{g} / \mathrm{dL}))$.

\section{Statistical analyses}

Data were presented as proportions for categorical variables and mean \pm standard deviation (SD) or median [interquartile range (IQR)] for continuous variables. Comparisons between HD and PD patients were made using $t$ - test or Wilcoxon rank-sum test for continuous variables, and chi-square test for categorical variables.

According to the K/DOQI guideline [8], patients with corrected serum calcium from 8.4 to $9.5 \mathrm{mg} / \mathrm{dL}$ ( 2.10 to $2.37 \mathrm{mmol} / \mathrm{L}$ ), phosphate from 3.5 to $5.5 \mathrm{mg} / \mathrm{dL}$ (1.13 to $1.78 \mathrm{mmol} / \mathrm{L})$ and $\mathrm{PTTH}$ from 150 to $300 \mathrm{pg} / \mathrm{mL}(150$ to $300 \mathrm{ng} / \mathrm{L}$ ) were considered optimal. The percentage of participants within the optimal range and outside the optimal range (above or below) was compared with the results of Dialysis Outcomes and Practice Study (DOPPS) 3 (2007) and DOPPS 4 (2010) [9].

Factors associated with hyperphosphatemia were analyzed using Logistic regression models. Factors in the models included age (continuous), sex (female vs male), dialysis pattern (PD vs HD), serum albumin level (continuous), hemoglobin level (continuous), and region of residency (south vs. north).

All analyses were performed by SPSS statistical package, version 16.0 (SPSS, Inc., Chicago, IL). A P value of less than 0.05 is considered statistically significant.

\section{Results}

Altogether 2074 patients were recruited, including 1711 HD patients and 363 PD patients. The average age was $57.5 \pm 14.3$ years, and $53.8 \%$ were males. The mean vintage of $\mathrm{HD}$ and $\mathrm{PD}$ were $47.8 \pm 41.2$ months and $24.1 \pm 20.0$ months, respectively. Compared with HD patients, PD patients were older, and had higher percentage of females. The main cause of ESRD was glomerulonephritis (47.5\%), while the percentage of diabetic nephropathy was higher among PD patients compared with HD patients (22.0\% vs $8.9 \%$ ) (Table 1$)$.

Only $38.5 \%, 39.6 \%$ and $26.6 \%$ of participants were within the optimal level of serum $\mathrm{P}$, corrected serum $\mathrm{Ca}$ and iPTH. There were no significant differences in percentages of achievement of optimal target in plasma P, Ca and iPTH between HD and PD patients. Serum P level and percentage of patients with hyperphosphatemia were statistically higher in the HD patients compared with those of PD patients, which was $6.3 \pm 2.1 \mathrm{mg} / \mathrm{dL}$ vs $5.7 \pm 2.0 \mathrm{mg} /$ $\mathrm{dL}(\mathrm{P}<0.001)$ and $57.4 \%$ vs $47.4 \%(\mathrm{P}<0.01)$, respectively. 


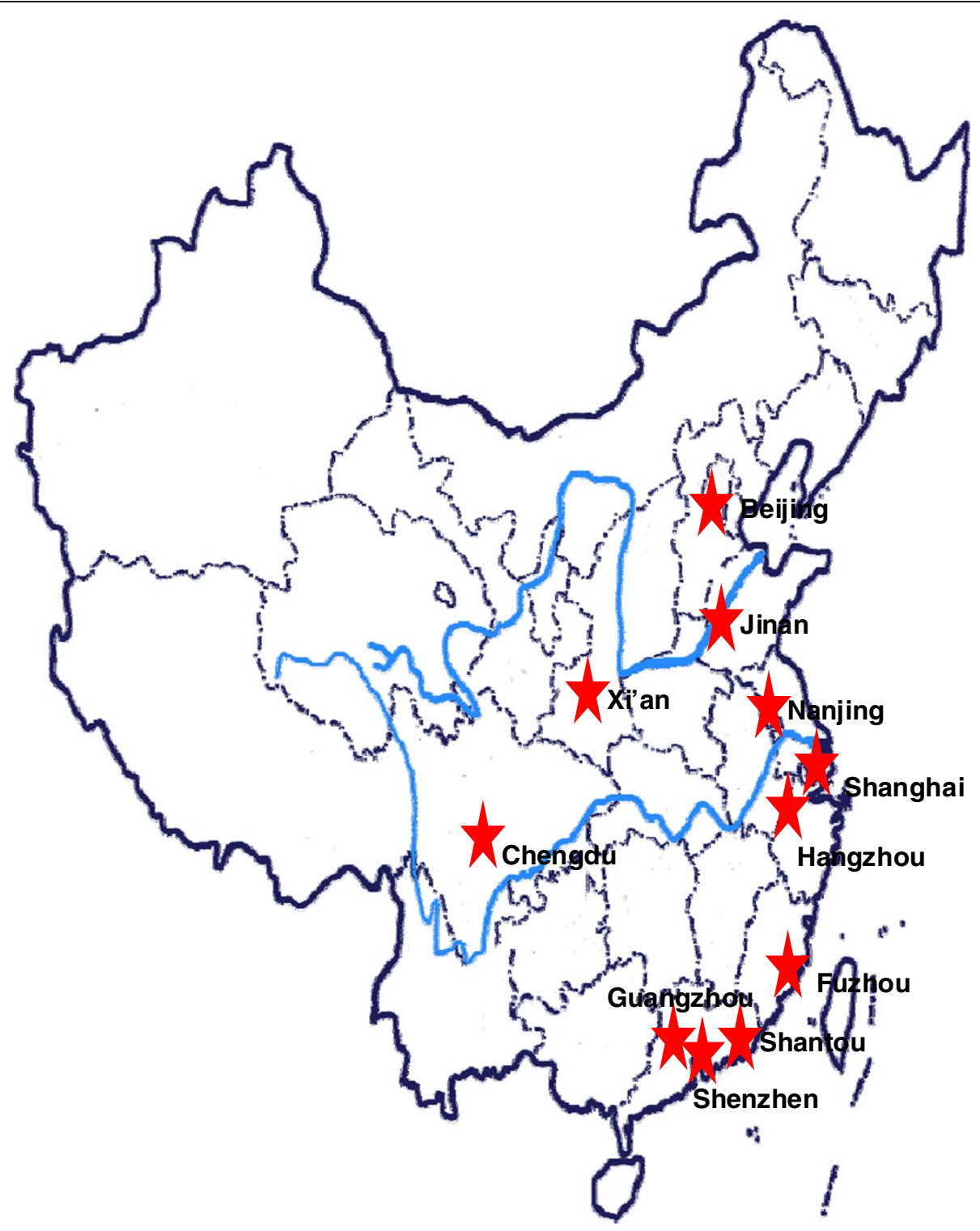

Figure 1 A map marked with study centers.

Serum Ca level was higher in HD patients compared with PD patients, but no significant differences in percentages of patients with hypercalcemia or hypocalcemia. Serum iPTH level were statistically higher in the PD patients compared with those of HD patients $(P=0.03)$. And the percentage of patients with low PTH was lower in PD patients compared with HD patients $(\mathrm{P}<0.001)$.

The percentages of patients with optimal $\mathrm{P}(37.6 \% \mathrm{vs}$ $49.8 \%$ vs $54.5 \%, \mathrm{P}<0.01)$, Ca $(38.6 \%$ vs $50.4 \%$ vs $56.0 \%$, $\mathrm{P}<0.01)$ and $\mathrm{iPTH}(26.5 \%$ vs $31.4 \%$ vs $32.1 \%, \mathrm{P}<0.01)$ were lower in HD patients, compared with those in the DOPPS 3 and DOPPS 4 (Table 2).

Treatment regimen regarding $\mathrm{MBD}$ was different between HD and PD patients. More patients in HD were prescribed with phosphorus binders than those of $\mathrm{PD}$ patients $(70.8 \%$ vs $64.0 \%, P=0.03)$. More HD patients were prescribed with vitamin $\mathrm{D}$ than PD patients $(69.2 \%$ vs $59.8 \%, \mathrm{P}<0.01)$. Compared with $\mathrm{HD}$ patients, the mean dose of elemental calcium intake was lower in PD patients $(1.11 \pm 0.67 \mathrm{~g} /$ day vs $0.91 \pm 0.52 \mathrm{~g} /$ day, $\mathrm{P}<0.01)$, and fewer patients exceeded the $1.5 \mathrm{~g} /$ day limit suggested by K/DOQI guidelines $(30.4 \%$ vs $19.5 \%, \mathrm{P}<0.01)$. All phosphorus binders used in those patients were calcium-based binders. Among patients with serum phosphorus level above $5.5 \mathrm{mg} / \mathrm{dL}, 27.4 \%$ of $\mathrm{HD}$ patients and $25.0 \%$ of PD patients were not receiving any phosphorus binder. Actually in our study, 98\% of patients who were prescribed vitamin D used calcitriol.

Serum P level and percentage of patients with hyperphosphatemia were statistically higher in the South region 
Table 1 Characteristics of the participants and comparison between HD and PD

\begin{tabular}{|c|c|c|c|c|}
\hline Variables & All participants $(n=2074)$ & $\mathrm{HD}(n=1711)$ & PD $(n=363)$ & $P$ value \\
\hline Age (years) & $57.5 \pm 14.3$ & $57.1 \pm 14.2$ & $59.0 \pm 14.4$ & 0.03 \\
\hline Male (\%) & 53.8 & 55.8 & 44.1 & $<0.001$ \\
\hline Duration of dialysis (months) & $33.6 \pm 40.7$ & $47.8 \pm 41.2$ & $24.1 \pm 20.0$ & $<0.001$ \\
\hline \multicolumn{5}{|l|}{ Cause of ESRD (\%) } \\
\hline Glomerulonephritis & 47.5 & 49.4 & 38.6 & $<0.001$ \\
\hline Hypertension & 12.1 & 11.6 & 14.6 & 0.11 \\
\hline Diabetes & 11.2 & 8.9 & 22.0 & $<0.001$ \\
\hline Interstitial nephritis & 7.4 & 7.6 & 6.3 & 0.44 \\
\hline Obstructive nephropathy & 2.4 & 2.4 & 2.5 & 0.85 \\
\hline Others & 19.4 & 20.1 & 16.0 & 0.18 \\
\hline Hemoglobin (g/L) & $101.0 \pm 2.1$ & $101.1 \pm 2.0$ & $99.3 \pm 2.1$ & 0.14 \\
\hline Serum albumin (mg/L) & $37.5 \pm 5.0$ & $37.8 \pm 4.8$ & $36.1 \pm 5.7$ & $<0.001$ \\
\hline \multicolumn{5}{|l|}{ Corrected calcium (mg/dL) (\%) } \\
\hline Mean \pm SD & $9.3 \pm 1.1$ & $9.3 \pm 1.1$ & $9.2 \pm 1.1$ & 0.03 \\
\hline$<8.4$ & 19.4 & 19.4 & 19.4 & 1.00 \\
\hline $8.4-9.5$ & 39.6 & 38.6 & 44.0 & 0.07 \\
\hline$>9.5$ & 41.0 & 42.0 & 36.6 & 0.06 \\
\hline Phosphorus binder prescription (\%) & 70.5 & 71.4 & 66.7 & 0.35 \\
\hline Vitamin D prescription (\%) & 61.2 & 64.6 & 43.2 & $<0.001$ \\
\hline \multicolumn{5}{|l|}{ Phosphorus (mg/dL) (\%) } \\
\hline Mean \pm SD & $6.2 \pm 2.1$ & $6.3 \pm 2.1$ & $5.7 \pm 2.0$ & $<0.001$ \\
\hline$<3.5$ & 5.8 & 5.0 & 9.6 & $<0.01$ \\
\hline $3.5-5.5$ & 38.5 & 37.6 & 43.0 & 0.06 \\
\hline$>5.5$ & 55.6 & 57.4 & 47.4 & $<0.01$ \\
\hline Phosphorus binder prescription (\%) & 73.0 & 72.6 & 75.0 & 0.65 \\
\hline Vitamin D prescription (\%) & 66.6 & 66.5 & 67.5 & 0.85 \\
\hline \multicolumn{5}{|l|}{ iPTH (pg/mL) (\%) } \\
\hline Median (IQR) & $269.0(132.0-472.9)$ & $265.0(127.2-456.3)$ & $304.5(162.0-486.7)$ & 0.03 \\
\hline$<150$ & 27.8 & 29.0 & 22.3 & $<0.01$ \\
\hline Vitamin D prescription (\%) & 48.1 & 52.3 & 23.5 & $<0.001$ \\
\hline $150-300$ & 26.6 & 26.5 & 27.3 & 0.74 \\
\hline Vitamin D prescription (\%) & 67.9 & 72.4 & 49.5 & $<0.001$ \\
\hline$>300$ & 45.5 & 44.5 & 50.4 & 0.05 \\
\hline Vitamin D prescription (\%) & 78.2 & 77.5 & 81.2 & 0.34 \\
\hline Phosphorus binder prescription (\%) & 69.4 & 70.8 & 64.0 & 0.03 \\
\hline Vitamin D prescription (\%) & 67.4 & 69.2 & 59.8 & $<0.01$ \\
\hline \multicolumn{5}{|l|}{ Dialysate calcium (\%) } \\
\hline $1.25 \mathrm{mmol} / \mathrm{L}$ & 19.0 & 19.9 & 15.1 & 0.08 \\
\hline $1.5 \mathrm{mmol} / \mathrm{L}$ & 58.2 & 68.7 & 12.8 & $<0.001$ \\
\hline $1.75 \mathrm{mmol} / \mathrm{L}$ & 22.8 & 11.4 & 72.1 & $<0.001$ \\
\hline Elemental calcium (g/day) & $1.07 \pm 0.65$ & $1.11 \pm 0.67$ & $0.91 \pm 0.52$ & $<0.001$ \\
\hline > 1.5 g/day (\%) & 28.4 & 30.4 & 19.5 & $<0.01$ \\
\hline
\end{tabular}

Abbreviations: $H D$, haemodialysis; $P D$, peritoneal dialysis; $E S R D$, end-stage renal disease; IQR, interquartile range; SD, standard deviation. 
Table 2 Comparison of HD and DOPPS 3 and DOPPS 4 in mineral metabolism laboratory parameters

\begin{tabular}{|c|c|c|c|}
\hline & $\mathrm{HD}(\mathrm{n}, \%)$ & DOPPS 3 (n, \%) & DOPPS $4(n, \%)$ \\
\hline \multicolumn{4}{|c|}{ Corrected calcium(mg/dL) } \\
\hline$<8.4$ & $324(19.4)^{*}$ & $791(12.5)$ & $904(12.4)$ \\
\hline $8.4-9.5$ & $647(38.6)^{*}$ & $3195(50.4)$ & $4080(56.0)$ \\
\hline$>9.5$ & $703(42.0)^{*}$ & $2355(37.1)$ & $2300(31.6)$ \\
\hline \multicolumn{4}{|c|}{ Phosphorus (mg/dL) } \\
\hline$<3.5$ & $86(5.0)^{*}$ & $765(11.0)$ & $895(11.3)$ \\
\hline $3.5-5.5$ & $642(37.6)^{*}$ & $3476(49.8)$ & $4337(54.5)$ \\
\hline$>5.5$ & $981(57.4)^{*}$ & $2738(39.2)$ & $2722(34.2)$ \\
\hline \multicolumn{4}{|c|}{ iPTH (pg/mL) } \\
\hline$<150$ & $473(29.0)^{*}$ & $2073(36.8)$ & $2325(32.5)$ \\
\hline $150-300$ & $431(26.5)^{*}$ & 1767 (31.4) & $2299(32.1)$ \\
\hline$>300$ & $724(44.5)^{*}$ & 1787 (31.8) & 2527 (35.3) \\
\hline
\end{tabular}

Abbreviations: HD, haemodialysis; DOPPS, Dialysis Outcomes and Practice Study.

${ }^{*} \mathrm{P}<0.01$ (HD group vs. DOPPS 3 and DOPPS 4).

DOPP 3(2007) and DOPP4 (2010): Includes data from AusNZ, Belgium, Canada, Germany, Italy, Japan, Spain, Sweden, UK, and US only.

patients compared with those of North region patients, which was $6.7 \pm 2.2 \mathrm{mg} / \mathrm{dL}$ vs $5.7 \pm 1.8 \mathrm{mg} / \mathrm{dL}(\mathrm{P}<0.001)$ and $65.8 \%$ vs $45.6 \%(\mathrm{P}<0.01)$, respectively. To the best of our knowledge, the availability of phosphorus binders or vitamin $\mathrm{D}$ is not different in north and south China. And in our study, $70.5 \%$ patients in South region and 68.4\% patients in North region were receiving phosphorus binder $(P=0.41)$. But more patients in North region were prescribed with vitamin $D$ than those patients in South region $(72.3 \%$ vs $62.7 \%, \mathrm{P}=0.045)$.

Table 3 presents the logistic regression analysis of hyperphosphatemia and various parameters. After adjusting for sex, serum albumin and hemoglobin, age, dialysis patterns and region of residency were still independently associated with hyperphosphatemia.

Table 3 The logistic regression analysis for hyperphosphatemia with different variables

\begin{tabular}{lll}
\hline Variables & $\begin{array}{l}\text { Crude OR } \\
\mathbf{( 9 5 \% ~ C l )}\end{array}$ & $\begin{array}{l}\text { Multivariable } \\
\text { adjusted } \\
\text { OR* } \mathbf{9 5 \% ~ C l )}\end{array}$ \\
\hline Age & $0.99(0.98-0.99)$ & $0.99(0.98-0.99)$ \\
Sex (female vs. male) & $0.82(0.69-0.99)$ & $0.88(0.73-1.07)$ \\
Dialysis patterns (PD vs. HD) & $0.72(0.57-0.92)$ & $0.74(0.57-0.95)$ \\
Serum albumin & $1.00(0.98-1.01)$ & $1.00(0.98-1.02)$ \\
Hemoglobin & $1.00(0.99-1.00)$ & $1.00(1.00-1.01)$ \\
region of residency & $2.14(1.78-2.57)$ & $2.15(1.77-2.62)$ \\
(South vs. North) & & \\
\hline
\end{tabular}

$\mathrm{OR}^{*}$ was adjusted for age, sex, dialytic patterns, serum albumin, hemoglobin, region of residency.

Abbreviations: $O R$, odds ration; $C l$, confidence interval.

\section{Discussion}

In our cross-sectional survey involving various geographic regions in China, the treatment of MBD was found to be sub-optimal among patients receiving mountainous dialysis. Uncontrolled hyperphosphatemia is especially prominent.

Hyperphosphatemia is an independent risk factor for both mortality $[1,10,11]$ and renal function decline in CKD patients [12,13]. A recent analysis of DOPPS [7] indicated that the lowest mortality was seen among patients with serum phosphorus 3.6 to $5.0 \mathrm{mg} / \mathrm{dL}$, and higher mortality was observed in facilities with a higher proportion of patients with serum phosphorus greater than $6.0 \mathrm{mg} / \mathrm{dL}$. Similarly, a meta-analysis indicated that the risk of death increased by $18 \%$ for every $1.0 \mathrm{mg} / \mathrm{dL}$ increase in serum phosphorus (relative risk of $1.18,95 \% \mathrm{CI}$ 1.12-1.25) in individuals with CKD [3]. Compared with results from DOPPS, control of hyperphosphatemia in our study is sub-optimal, which may be related to the following factors. Firstly, restriction of dietary phosphorus is the primary strategy for controlling hyperphosphatemia. Unfortunately, nutritionist was not available for most HD and PD centers in China. In our analysis, although we did not have information of dietary intake of phosphorus, residency in southern China was observed to be an independent risk factor for hyperphosphatemia. It is well known that in southern China, foods containing rich phosphorus (eg. seafood and meat soup) are more likely to be consumed than in Northern China. A study using 24-hour dietary recalls also indicated that phosphorus intake was higher among Southern Chinese compared with that of Northern Chinese, which was $562 \pm 67 \mathrm{mg} /$ $1000 \mathrm{kcal}$ and $378 \pm 76 \mathrm{mg} / 1000 \mathrm{kcal}$, respectively [14]. Secondly, insufficient use of phosphorus binder was observed in our analysis. On the other hand, $46.5 \%$ of patients with serum calcium level above $9.5 \mathrm{mg} / \mathrm{dL}$ still were using calcium-containing phosphorus binder. In China, non-containing calcium phosphorus binders were not available. Therefore, inappropriate application of phosphorus binder may be related to hyperphosphatemia. Finally, our analysis revealed that HD was independently associated with higher risk of hyperphosphatemia, compared with PD patients. It has been shown that certain hemodialysis prescription may increase phosphorus clearance. Nocturnal HD [15,16] and short daily HD [17,18] was shown to reduce serum phosphorus levels and requirement for phosphorus binders. Membrane surface area itself has a potentially important impact on phosphorus removal. In a recent study in 18 patients over a period of 6 weeks, doubling of membrane area by the use of 2 dialyzers in parallel (with blood flow equally split between the dialyzers) resulted in a $1.34 \mathrm{mg} / \mathrm{dL}$ decline in predialysis serum phosphorus levels compared with conventional HD [19]. A recent randomized controlled trial 
evaluated the effect of different hemodialysis prescription on hyperphosphatemia among 493 patients [20]. It was shown that phosphorus levels decreased from $5.18 \pm 0.10 \mathrm{mg} / \mathrm{dL}$ at baseline to $4.87 \pm 0.10 \mathrm{mg} / \mathrm{dL}$ at 6 months in hemodiafiltration patients $(\mathrm{P}<0.001)$ and were stable in low-flux HD patients $(5.10 \pm 0.10 \mathrm{mg} / \mathrm{dL}$ at baseline and $5.03 \pm 0.10 \mathrm{mg} / \mathrm{dL}$ after 6 months, $\mathrm{P}=0.5$ ). After adjustment for phosphorus binder use, hemodiafiltration still have an advantage of improving phosphorus control over low-flux HD. In our study, low-flux HD was applied in the majority of our dialysis centers, which may also be related to poor phosphorus control. In summary, reducing phosphorus intake under the instruction of nutritionist, proper use of phosphorus binder, and adopting dialysis prescription with better phosphorus clearance may improve the sub-optimal treatment of hyperphosphatemia in China.

In K/DOQI guidelines, the optimal PTH levels was recommended to be $150-300 \mathrm{pg} / \mathrm{ml}$ for dialysis patients, which was based on the predictive ability of PTH for low-and high-turnover bone disease [8]. However, the early studies of MBD parameters evaluated survival as a function of a single time point baseline measurement of $\mathrm{PTH}$, thereby ignoring important changes in the parameter [11]. Conversely, time dependent approaches are influenced strongly by events immediately proximal to the outcome and therefore may reflect worsening parameters caused by rapidly declining health status $[10,21]$. More recently, there have been attempts at using cumulative-effects models by the Hemodialysis (HEMO) Study investigators to better capture the relationship between bone mineral metabolism parameters and survival, and they also have found higher ceilings for PTH levels than previous work [22]. Analysis from the DOPPS also revealed an increased risk for all-cause mortality (not cardiovascular mortality) only when $\mathrm{PTH}>600 \mathrm{pg} / \mathrm{mL}$ [7]. Therefore, in the recently released guidelines from the kidney Disease: Improving Global Outcomes (KDIGO), it is suggested that iPTH levels should be maintained in the range of approximately two to nine times the upper limit of normal for patients receiving dialysis [23]. In our study, about $56.7 \%$ of participants met the KDIGO target for PTH. However, more studies (especially interventional studies) are needed to verify the range suitable for better clinical outcome. In our study, the proportion of PD patients with PTH less than $150 \mathrm{pg} / \mathrm{mL}$ is not higher than HD patients. This may be related to the relatively low proportion of diabetes in PD patients and with short time on dialysis.

To date, there is no DOPPS-equivalent study looked at the management of mineral metabolism in PD patients. Compared with the data from Canadian PD patients [24], the present study demonstrated that the percentage of patients reached the K/DOQI targets for
Ca (44.0\% vs $44.7 \%)$ and iPTH (27.3\% vs $28.4 \%)$ were similar among PD patients. However, the percentage of patients with hyperphosphatemia (> $5.5 \mathrm{mg} / \mathrm{dL}$ ) and hypercalcemia (> $9.5 \mathrm{mg} / \mathrm{dL}$ ) were higher than Canadian PD patients, which were $47.4 \%$ vs $21.2 \%$ and $36.6 \%$ vs $16.7 \%$, respectively. It indicates that based on K/DOQI guidelines, Chinese PD patients are not within optimal target ranges and there is room for improvement.

Our study has several limitations that deserve mention. Firstly, study sites were enrolled on a voluntary basis, which might introduce bias. However, most of participants came from tertiary hospitals. Therefore, the direction of bias would be to overestimate the control rate of MBD. Secondly, some information of factors related to the MBD were not available in our study, such as diet, residual renal function, dialysis prescription and dialysis adequacy. Thirdly, laboratory tests were not performed in one central laboratory, therefore variability of measurement may affect the interpretation of our results. The same limitation exists for the comparison between our study and DOPPS study. Fourthly, the demographic characteristics and medical conditions of patients in our study might be different from those of participants in DOPPS study, which might contribute to the difference in parameters of MBD. Finally, the cross-sectional design limited our ability to make causal inference.

\section{Conclusion}

Our national survey indicated that the status of MBD treatment is sub-optimal among Chinese patients receiving dialysis. The issue of hyperphosphatemia is prominent and needs to be improved. Multiple measures including dietary phosphorus restriction, oral phosphorus binders including non-containing calcium phosphorus binders and dialysis model should be optimized to improving the control of hyperphosphatemia, which in turn may reduce the risk for vascular calcification and cardiovascular mortality.

\section{Competing interests}

The authors declare that they have no competing interests.

\section{Author's contributions}

$\mathrm{KXL}$ and $\mathrm{Z} \mathrm{LX}$ participated in the study, analyzed the data, interpreted the results, and drafted the manuscript. $Z L, C N, G Y, Y X Q, L W H, C J H, P L R, Y$ WJ, W H, C W, F MH, H LQ, D F, C XM, X ZY, Z JY, J Q, S W, X CY, T XL, H FF, $S$ GY, M CL, W L, X DM and N ZH participated in the survey and study design and collected the data. Z L participated in the survey and study design and interpreted the results. W M formed the study concept, interpreted the results, and revised the manuscript. Wang HY revised the manuscript for important intellectual content. All authors read and approved the final manuscript.

\section{Acknowledgments}

We would like to acknowledge The Roche Pharma. for generous sponsorship. 


\section{Author details}

${ }^{1}$ Renal Division, Department of Medicine, Peking University First Hospital; Peking University Institute of Nephrology; Key Laboratory of Renal Disease, Ministry of Health of China; Key Laboratory of Chronic Kidney Disease Prevention and Treatment (Peking University), Ministry of Education, Beijing, China. ${ }^{2}$ Department of Nephrology, China-Japan Friendship Hospital, Beijing, China. ${ }^{3}$ Department of Nephrology, Ruijin Hospital, Shanghai Jiao Tong University, School of Medicine, Shanghai, China. ${ }^{4}$ Renal Division, Xinhua Hospital, Shanghai Jiao Tong University, School of Medicine, Shanghai, China. ${ }^{5}$ Department of Nephrology, the First Affiliated Hospital, Sun Yat-sen University, Guangzhou, China. ${ }^{6}$ Department of Nephrology, Beijing Friendship Hospital, Capital Medical University, Beijing, China. ${ }^{7}$ Kidney Diseases Center, First Affiliated Hospital, Zhejiang University School of Medicine, Hangzhou, China. ${ }^{8}$ Department of Nephrology, Beijing Chaoyang Hospital, Capita Medical University, Beijing, China. ${ }^{9}$ Department of Nephrology, Shanghai First People s Hospital, Shanghai Jiaotong University, Shanghai, China.

${ }^{10}$ Department of Nephrology, Beijing Hospital, Beijing, China. ${ }^{11}$ Department of Nephrology, Xijing Hospital, Fourth Military Medical University, Xi'an, China. ${ }^{12}$ Department of Nephrology, Peking University Third Hospital, Beijing, China. ${ }^{13}$ Department of Nephrology, Shuguang Hospital, Shanghai University of Traditional Chinese Medicine, Shanghai, China. ${ }^{14}$ Department of Nephrology, Huashan Hospital, Fudan University, Shanghai, China.

${ }^{15}$ Department of Nephrology, The General Hospital of the People's Liberation Army, Beijing, China. ${ }^{16}$ Renal Division, Hospital Peking of Shenzhen, Shenzhen, China. ${ }^{17}$ Department of Nephrology, The 455th Hospital of PLA, Shanghai, China. ${ }^{18}$ Department of Nephrology, Xuanwu Hospital, Capital Medical University, Beijing, China. ${ }^{19}$ Department of Nephrology, Guangdong General Hospital, Guangzhou, China. ${ }^{20}$ Renal Division, Jiangsu Provincial Hospital, Nanjing, China. ${ }^{21}$ Department of internal medicine, Shantou Central Hospital, Shantou, China. ${ }^{22}$ Department of Nephrology, Nanfang Hospital, The Southern Medical University, Guangzhou, China. ${ }^{23}$ Department of Nephrology, Fujian Provincial Hospital, Fuzhou, China. ${ }^{24}$ Department of Nephrology, Changzheng Hospital, Second Military Medical University, Shanghai, China. ${ }^{25}$ Department of Nephrology, Sichuan Provincial People' s Hospital, Chengdu, China. ${ }^{26}$ Department of Nephrology, Qianfoshan Hospital, Shandong University, Jinan, China. ${ }^{27}$ Renal Division, Renji Hospital, Shanghai Jiao Tong University School of Medicine, Shanghai, China. ${ }^{28}$ Department of Nephrology, Peking University People's Hospital, Beijing, China. ${ }^{29}$ Institute of Nephrology and Division of Nephrology, and Key Laboratory of Ministry of Health, Peking University First Hospital, 8 Xishiku Street, Xicheng District, Beijing 100034, China.

Received: 13 December 2011 Accepted: 14 September 2012 Published: 21 September 2012

\section{References}

1. Block GA, Klassen PS, Lazarus JM, Ofsthun N, Lowrie EG, Chertow GM: Mineral metabolism, mortality, and morbidity in maintenance hemodialysis. J Am Soc Nephrol 2004, 15(8):2208-2218.

2. Hruska KA, Saab G, Mathew S, Lund R: Renal osteodystrophy, phosphate homeostasis, and vascular calcification. Semin Dial 2007, 20(4):309-315.

3. Palmer SC, Hayen A, Macaskill P, et al: Serum levels of phosphorus, parathyroid hormone, and calcium and risks of death and cardiovascular disease in individuals with chronic kidney disease: a systematic review and meta-analysis. JAMA 2011, 305(11):1119-1127.

4. Levin A, Bakris GL, Molitch $M$, et al: Prevalence of abnormal serum vitamin D, PTH, calcium, and phosphorus in patients with chronic kidney disease: results of the study to evaluate early kidney disease. Kidney Int 2007, 71(1):31-38.

5. Gutierrez O, Isakova T, Rhee $\mathrm{E}$, et al: Fibroblast growth factor-23 mitigates hyperphosphatemia but accentuates calcitriol deficiency in chronic kidney disease. J Am Soc Nephrol 2005, 16(7):2205-2215.

6. Wald R, Tentori F, Tighiouart H, Zager PG, Miskulin DC: Impact of the Kidney Disease Outcomes Quality Initiative (KDOQI) Clinical Practice Guidelines for Bone Metabolism and Disease in a large dialysis network. Am J Kidney Dis 2007, 49(2):257-266.

7. Tentori F: Mineral and bone disorder and outcomes in hemodialysis patients: results from the DOPPS. Semin Dial 2010, 23(1):10-14.

8. KVOQI clinical practice guidelines for bone metabolism and disease in chronic kidney disease: Am J Kidney Dis 2003, 42 (4 Suppl 3):S1-S201.
9. Dialysis Outcomes and Practice Patterns Study: DOPPS annual report. 2010 http//www.dopps.org/annual report/index.htm.

10. Kalantar-Zadeh K, Kuwae N, Regidor DL, et al: Survival predictability of time-varying indicators of bone disease in maintenance hemodialysis patients. Kidney Int 2006, 70(4):771-780.

11. Block GA, Hulbert-Shearon TE, Levin NW, Port FK: Association of serum phosphorus and calcium $\mathrm{x}$ phosphate product with mortality risk in chronic hemodialysis patients: a national study. Am J Kidney Dis 1998, 31(4):607-617

12. Schwarz S, Trivedi BK, Kalantar-Zadeh K, Kovesdy CP: Association of disorders in mineral metabolism with progression of chronic kidney disease. Clin J Am Soc Nephrol 2006, 1(4):825-831.

13. Kovesdy CP, Kalantar-Zadeh K: Serum phosphorus and the risk of progression of chronic kidney disease. Nephrol Dial Transplant 2007, 22(12):3679-3680.

14. Zhao L, Stamler J, Yan LL, et al: Blood pressure differences between northern and southern Chinese: role of dietary factors: the International Study on Macronutrients and Blood Pressure. Hypertension 2004, 43(6):1332-1337.

15. Walsh M, Manns BJ, Klarenbach S, Tonelli M, Hemmelgarn B, Culleton B: The effects of nocturnal compared with conventional hemodialysis on mineral metabolism: A randomized-controlled trial. Hemodial Int 2010, 14(2):174-181

16. Pierratos A, Ouwendyk M, Francoeur R, et al: Nocturnal hemodialysis: three-year experience. J Am Soc Nephrol 1998, 9(5):859-868.

17. Ayus JC, Mizani MR, Achinger SG, Thadhani R, Go AS, Lee S: Effects of short daily versus conventional hemodialysis on left ventricular hypertrophy and inflammatory markers: a prospective, controlled study. J Am SoC Nephrol 2005, 16(9):2778-2788.

18. Maduell F, Navarro $V$, Torregrosa $E$, et al: Change from three times a week on-line hemodiafiltration to short daily on-line hemodiafiltration. Kidney Int 2003, 64(1):305-313.

19. Tonelli M, Wang W, Hemmelgarn B, Lloyd A, Manns B: Phosphate removal with several thrice-weekly dialysis methods in overweight hemodialysis patients. Am J Kidney Dis 2009, 54(6):1108-1115.

20. Penne EL, van der Weerd NC, van den Dorpel MA, et al: Short-term effects of online hemodiafiltration on phosphate control: a result from the randomized controlled Convective Transport Study (CONTRAST). Am J Kidney Dis 2010, 55(1):77-87.

21. Melamed ML, Eustace JA, Plantinga $L$, et al: Changes in serum calcium, phosphate, and PTH and the risk of death in incident dialysis patients: a longitudinal study. Kidney Int 2006, 70(2):351-357.

22. Wald R, Sarnak MJ, Tighiouart $H$, et al: Disordered mineral metabolism in hemodialysis patients: an analysis of cumulative effects in the Hemodialysis (HEMO) Study. Am J Kidney Dis 2008, 52(3):531-540.

23. KDIGO clinical practice guideline for the diagnosis, evaluation, prevention, and treatment of Chronic Kidney Disease-Mineral and Bone Disorder (CKD-MBD): Kidney Int Supp/ 2009, 76(113):S1-S130.

24. Soroka SD, Beard KM, Mendelssohn DC, Cournoyer SH, Da Roza GA, Geary DF: Mineral metabolism management in Canadian peritoneal dialysis patients. Clin Nephrol 2011, 75(5):410-415.

doi:10.1186/1471-2369-13-116

Cite this article as: Kong et al:: Mineral and bone disorder in Chinese dialysis patients: a multicenter study. BMC Nephrology 2012 13:116.

\section{Submit your next manuscript to BioMed Central and take full advantage of:}

- Convenient online submission

- Thorough peer review

- No space constraints or color figure charges

- Immediate publication on acceptance

- Inclusion in PubMed, CAS, Scopus and Google Scholar

- Research which is freely available for redistribution 